\title{
Klinik-MVZs wirtschaftlich kein Selbstläufer
}

Medizinische Versorgungszentren in Klinikhand: Das gilt als vielversprechende Strategie für stationäre Einrichtungen, um sich Patienteneinweisungen zu sichern. Bei der Rentabilität gibt es allerdings enorme Unterschiede. MVZs zu betreiben, gilt für Krankenhäuser weiter als „erhebliche Herausforderung“.

B etrachtet man die wirtschaftlichen Ergebnisse, die Kliniken mit ihren MVZs erzielen, wird klar, dass der Betrieb einer solchen ambulanten Einrichtung eine erhebliche Herausforderung für ein Krankenhaus darstellt", heißt es in einer aktuellen Expertise von Statis e.V., einer bundesweiten Plattform für den internen Erfahrungsaustausch klinikgetragener MVZs. Die durchschnittliche Umsatzrendite von Klinik-MVZs beträgt laut Statis nur 0,83 Prozent. Die Streuung ist dabei groß: So verbleiben in einzelnen MVZ bis zu 18 Prozent des Umsatzes als Betriebsgewinn; andere Häuser verbuchen Verluste von 30 Prozent des Umsatzes oder darüber.

\section{Leistungsspektrum ist entscheidend}

Für einen rentablen Betrieb von MVZs gibt es der Expertise zufolge bestimmte Grundfaktoren. So besteht ein direkter und starker Zusammenhang zwischen dem Leistungsspektrum eines MVZs und seiner Rentabilität. Ein wirtschaftlicher Betrieb ist mit einer Beschränkung auf das ärztliche $\mathrm{Ba}$ sisspektrum nur in wenigen Fachrichtungen erreichbar. Ein Klinik-MVZ als Basisversorger, der auf lukrative Operationen, Laboruntersuchungen und andere Spezialleistungen mit Rücksicht auf Einweiser oder Chefärzte verzichten muss, arbeitet mit einem strategischen Handicap, weil der mögliche Leistungsumfang hinsichtlich Fallzahl und Leistungsspektrum nicht ausgeschöpft werden kann. In diesen Fällen kommt es besonders auf

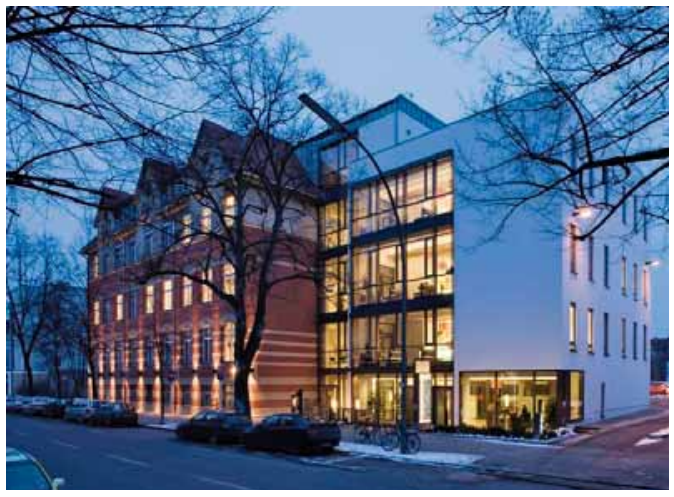

Poli(ti)kum: Das Berliner „Polikum“ wurde 2004 vom Allgemeinarzt Wolfram Otto gegründet - als erstes privat geführtes MVZ bundesweit. Polikum gilt als der bekannteste und einer der größten MVZ-Betreiber bundesweit. Ende 2014 wurde das Pionierunternehmen von der Sana Kliniken AG übernommen.

eine optimale betriebswirtschaftliche Steuerung des MVZ an, um Defizite zu vermeiden.

\section{Ärzte teurer als in Praxen}

Ein anderer wichtiger Einflussfaktor auf die Rentabilität eines KlinikMVZ sind naturgemäß die Gehaltskosten für die angestellten Ärzte. In Klinik-MVZ liegen laut Statis für die meisten Fachgruppen die Aufwendungen für den ärztlichen Dienst zwischen 50 Prozent und 75 Prozent des von den Ärzten generierten Umsatzes. Zu diesen Aufwendungen zählen Brutto-Gehälter, Sozialabgaben oder Zahlungen für Personalüberlassung an das Krankenhaus. Betrachtet man als Referenz die durchschnittlichen Betriebskosten in den Praxen niedergelassener Ärzte (für die meisten Fachrichtungen um die 50 Prozent des Umsatzes), so liegen die zuvor genannten Ausgaben für den ärztlichen Dienst in Klinik-MVZ zu hoch. Denn wenn sich Betriebskosten und Arztgehälter rechnerisch auf über
100 Prozent des erzielten Umsatzes summieren, ist die betreffende Abteilung defizitär.

Eine personell gut ausgestattete MVZ-Verwaltung scheint sich gemäß der von Statis erhobenen Zahlen positiv auf die Rentabilität von KlinikMVZ auszuwirken. Zwar kostet die Verwaltung Geld. Doch scheint es so zu sein, dass bei entsprechender Ausrichtung der Verwaltung die ärztliche Leistung besser gesteuert und die Erträge erhöht werden können.

Anders als der Bundesverband Medizinische Versorgungszentren (BMVZ) versteht sich der Statis e.V. nicht als nach außen wirkender, politischer Interessenverband, sondern als institutionalisiertes Forum für den internen Erfahrungsaustausch von MVZs in Trägerschaft stationärer Einrichtungen. Knapp 30 Klinik-MVZs haben sich dem Statis e.V. seit seiner Gründung im Januar 2014 angeschlossen.

Adalbert Zehnder 